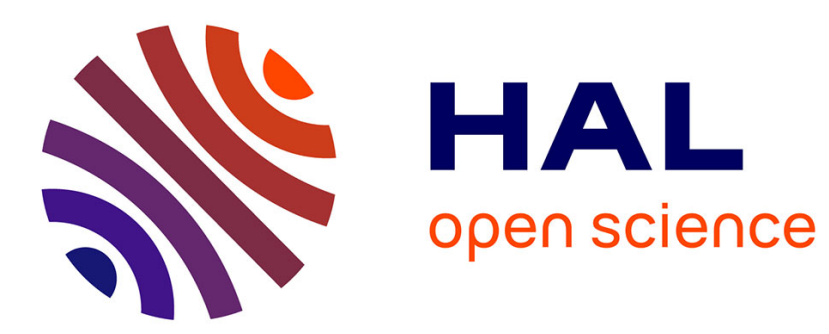

\title{
Mise en évidence d'un état magnétique non colinéaire dans les multicouches Fe/Gd
}

Ph. Bauer, M. Sajieddine, B. George, M. Vaezzadeh, C. Dufour, K. Cherifi, G. Marchal, Ph. Mangin

\section{- To cite this version:}

Ph. Bauer, M. Sajieddine, B. George, M. Vaezzadeh, C. Dufour, et al.. Mise en évidence d'un état magnétique non colinéaire dans les multicouches Fe/Gd. Journal de Physique IV Proceedings, 1992, 02 (C3), pp.C3-199-C3-203. 10.1051/jp4:1992329 • jpa-00251534

\section{HAL Id: jpa-00251534 https://hal.science/jpa-00251534}

Submitted on 1 Jan 1992

HAL is a multi-disciplinary open access archive for the deposit and dissemination of scientific research documents, whether they are published or not. The documents may come from teaching and research institutions in France or abroad, or from public or private research centers.
L'archive ouverte pluridisciplinaire $\mathbf{H A L}$, est destinée au dépôt et à la diffusion de documents scientifiques de niveau recherche, publiés ou non, émanant des établissements d'enseignement et de recherche français ou étrangers, des laboratoires publics ou privés. 


\title{
Mise en évidence d'un état magnétique non colinéaire dans les multicouches Fe/Gd
}

\author{
Ph. BAUER, M. SAJIEDDINE, B. GEORGE, M. VAEZZADEH, C. DUFOUR, K. CHERIFI, \\ G. MARCHAL et Ph. MANGIN
}

Laboratoire de Physique du Solide, BP. 239, 54506 Vandoeuvre cedex, France

\begin{abstract}
In the $\mathrm{Fe} / \mathrm{Gd}$ multilayered system the magnetic moments are constrained to be in the film plane owing to dominant shape anisotropy, provided the absence of crystal field effects upon the $S$ state of $\mathrm{Gd}^{3+}$. The in-plane magnetic structure versus an external field applied parallel to the plane is studied through magnetization, magnetoresistance measurements and ${ }^{57} \mathrm{Fe}$ Mössbauer spectroscopy. The transition from the "aligned state" to a "twisted state" is evidenced at a critical $\mathrm{H}^{*}$ field for a $\mathrm{Fe}(42 \AA) / \mathrm{Gd}(84 \AA)$ sample. It turns out that $\mathrm{H}^{*}$ depends upon temperature and respective $\mathrm{Fe}-\mathrm{Gd}$ submagnetizations. The "twisted state" is favoured at the compensation temperature.
\end{abstract}

\section{Introduction}

L'étude du magnétisme des couches minces Métal de Transition/Terre Rare s'est avérée féconde tant au niveau fondamental que pour les retombées technologiques (enregistrement magnétique perpendiculaire). La nature de la Terre Rare aussi bien que les paramètres structuraux (épaisseur individuelle, température du substrat lors de l'élaboration) jouent un rôle déterminant dans l'anisotropie magnétique [1][2][3]. De son côté, le système $\mathrm{Fe} / \mathrm{Gd}$ permet de s'affranchir des effets de champ cristallin (état $S$ de lion $\mathrm{Gd}^{3+}$ ) et de ne prendre en compte que les paramètres structuraux et les interactions d'échange Fe-Gd.

Les premières études expérimentales effectuées sur les multicouches $\mathrm{Fe} / \mathrm{Gd}$ montrent l'existence de températures de compensation, de transitions magnétiques et de variations brutales de la susceptibilité [4],[5],[6]. D'un point de vue théorique Camley et al. [7],[8] ont proposé des diagrammes d'états magnétiques dépendant du champ extérieur, des aimantations $\mathrm{Fe}, \mathrm{Gd}$ respectives et des épaisseurs relatives des couches. Avec les hyphotèses d'un couplage Fe-Gd antiparallèle à l'interface et d'intégrales d'échange analogues à celles des constituants massifs, ces auteurs montrent l'existence possible de quatre états magnétiques: i) l'état "Gd aligné" où les moments du gadolinium sont alignés dans le sens du champ magnétique et les moments du fer en sens inverse, ii) l'état "Fe aligné" avec les moments du fer alignés dans le sens du champ et ceux du gadolinium antiparallèles, iii) un état "Twisté" pour lequel l'angle entre le champ externe et les moments du fer et du gadolinium varie de façon continue, et, iv) un état "A1" avec des atomes de gadolinium paramagnétiques au coeur des couches. 
Dans cet article nous présentons un diagramme expérimental de phases magnétiques pour une multicouche $\mathrm{Fe}(42 \AA) / \mathrm{Gd}(84 \AA)$ dont l'aimantation du gadolinium est dominante à basse température. La transition de l'état aligné à l'état twisté est mise en évidence à partir de l'examen des isothermes $M(H)$ à diverses températures, par magnétorésistance et par spectrométrie Mössbauer $\left({ }^{57} \mathrm{Fer}\right)$ en utilisant une géométrie appropriée.

\section{Techniques expérimentales}

La multicouche est élaborée par évaporation sous vide $\left(10^{-8} \mathrm{Torr}\right)$ et condensation alternée sur un substrat maintenu à $90 \mathrm{~K}$. L'épaisseur des couches est controlée par des balances à quartz pilotées par ordinateur. L'erreur de l'épaisseur par couche est estimée à $\pm 3 \%$. Afin d'éviter l'oxydation du gadolinium, la dernière couche est une couche de fer recouverte de $200 \AA$ de silicium. Une description plus détaillée de l'élaboration peut être trouvée en [9]. L'échantillon est caractérisé par sa résistivité insitu pendant l'élaboration, par diffraction de rayons $X$ aux grands et petits angles et par microscopie électronique. Les mesures d'aimantation ont été effectuées de $4,2 \mathrm{~K}$ à $310 \mathrm{~K}$ dans un magnétomètre de type Foner pour des champs parallèles à l'échantillon jusqu'à 20 kOe et avec un S.Q.U.I.D. jusqu'à $50 \mathrm{kOe}$.

La spectrométrie Mössbauer utilisée avec une géométrie particulière [10] permet de déterminer à la fois l'orientation moyenne $\tilde{\alpha}$ et le sens des moments du fer par rapport à un champ magnétique $H_{\text {ext }}$ appliqué parallèlement au plan des couches. Les intensités relatives du sextuplet Zeeman sont dans les rapports $3: X: 1: 1: X: 3$ où $X$, relatif aux pics intermédiaires, est fonction de l'orientation des moments du fer par rapport à la direction des rayons $\gamma$ incidents (notée $\vec{\gamma}$ ). Quand tous les moments sont dans le plan de l'échantillon (perpendiculaire à $\vec{\gamma}$ ), on doit avoir $X=4$ quel que soit $H_{\text {ext }}$. Pour avoir accès à la distribution de l'orientation des moments du fer dans le plan de l'échantillon, il suffit de tourner ce dernier autour de l'axe du champ tel que l'angle entre la normale au plan et $\vec{\gamma}$ soit par exemple $45^{\circ}$ (au lieu de $0^{\circ}$ ). On obtient $X=4,1,33$ et 2,4 respectivement quand tous les moments sont alignés suivant $H_{\text {ext }}$, perpendiculaires à $H_{\text {ext }}$ et aléatoirement distribués dans le plan. De plus, quand les moments du fer sont antiparallèles à $H_{\text {ext }}$, le champ hyperfin $H_{\text {hyp }}\left(H_{\text {ext }}\right)$ existant au noyau de ${ }^{57} \mathrm{Fer}$ est augmenté par rapport à la valeur standard $H_{\text {hyp }}(0)$ et diminué pour un alignement parallèle.

\section{Résultats et discussion}

La résistivité d'un échantillon multicouche $\mathrm{Fe} / \mathrm{Gd}$ est essentiellement due à la résistivité des couches de fer pour des épaisseurs voisines de Fe et de $G d$ ( $\rho_{G d} \approx 6 \rho_{\mathrm{Fe}}$ à température ambiante). De plus, dans le cas des métaux de transition, la résistivité 
dépend de l'orientation relative du courant de mesure avec la direction des moments magnétiques. Cette dépendance est telle que $\rho_{/ /}>\rho_{\perp}$ où $\rho_{/ /}$et $\rho_{\perp}$ sont les résistivités mesurées lorsque les moments magnétiques sont respectivement parallèles et perpendiculaires à la direction du courant de mesure. La mise en évidence de la transition état aligné - état twisté se manifeste sur les spectres de magnétorésistance dans une configuration transverse (champ perpendiculaire au courant de mesure), par une brusque remontée de la résistance à partir d'un champ critique $H^{*}$ (Fig. 1) .

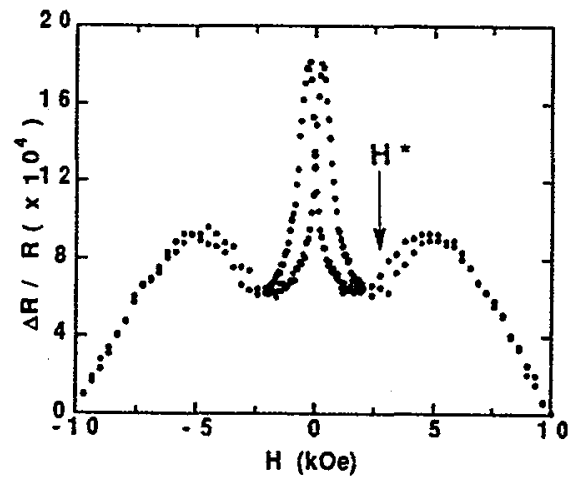

Fig. 1.-Magnétorésistance à $4,2 \mathrm{~K}$ dans une configuration transverse (champ magnétique et courant dans le plan des couches, perpendiculaires entre eux).

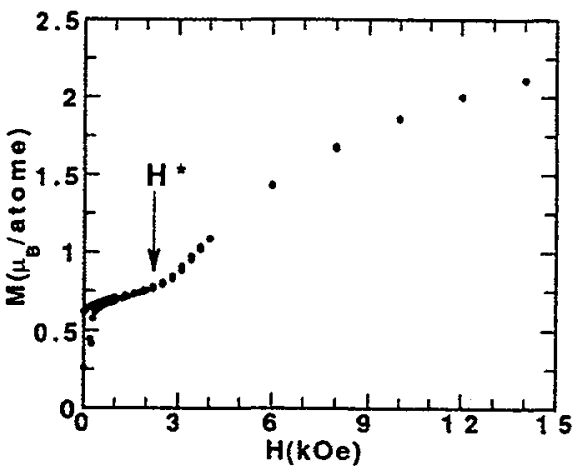

Fig.2.-Isotherme magnétique à $100 \mathrm{~K}$

L'évolution de l'aimantation en fonction du champ à $100 \mathrm{~K}$ (aimantation du $\mathrm{Gd}$ dominante) montre une brusque augmentation de la susceptibilité $\chi$ pour une certaine valeur $\mathrm{H}^{*}$ après un premier palier de saturation (fig. 2). En champ élevé l'aimantation tend à nouveau vers une saturation. A la température de compensation Tcp ce palier disparaît: on observe une faible valeur de $\mathrm{H}^{*}$ associée à une susceptibilité plus importante. Au dessus de Tcp le premier palier de saturation réapparaît. Les variations en fonction de $T$, de $\mathrm{H}^{*}$ et $\chi^{*}$ correspondantes sont reportées sur la figure 3 . La ligne $H^{*}(T)$ représente la transition entre l'état aligné et l'état twisté avec un minimum marqué au voisinage de Tcp, température pour laquelle on observe un pic de $\chi^{*}$. Ce diagramme est conforme au prévisions de Camley et al.; en particulier l'état twisté est favorisé quand les aimantations $\mathrm{Fe}$ et $\mathrm{Gd}$ respectives se compensent. 


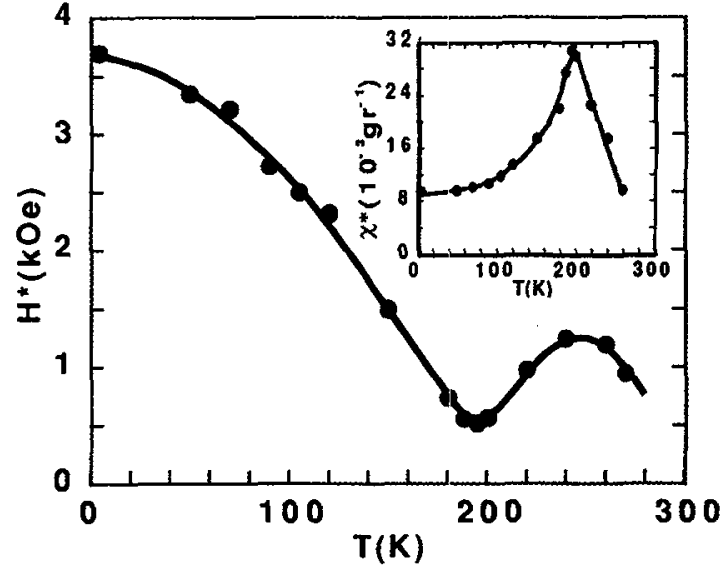

Fig. 3.-Variation de $\mathrm{H}^{*}$ et $\chi^{*}$ avec la température.

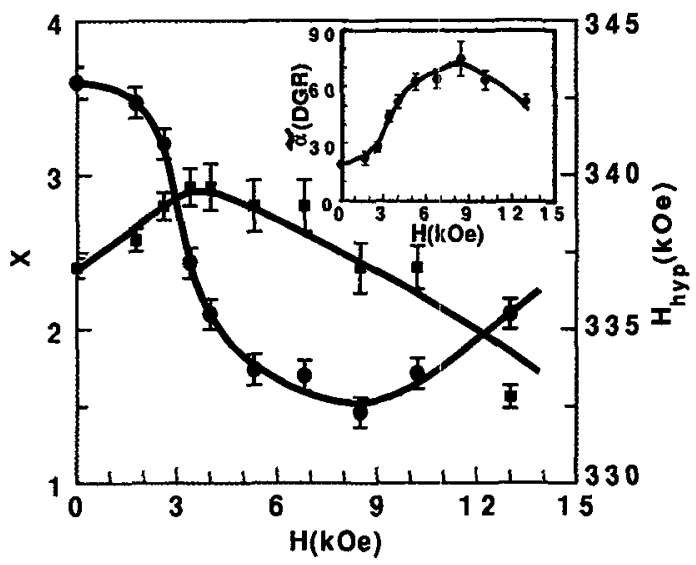

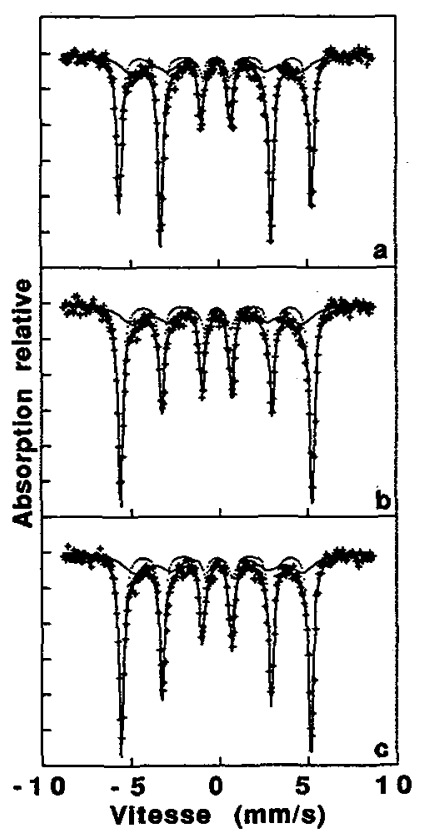

Fig. 4.-Spectres Mössbauer à $100 \mathrm{~K}$ (échantillon incliné) avec champ magnétique parallèle au plan des couches.

a) $0 \mathrm{KOe}, \mathrm{b}) 8,5 \mathrm{KOe}, \mathrm{c)} 13 \mathrm{KOe}$.

Fig. 5.-Variation de $\mathrm{X}, \mathrm{H}_{\text {hyp }}$ et $\tilde{\alpha}$ avec $\mathrm{H}$ le champ extérieur

La figure 4 montre trois spectres Mössbauer effectués à 100K avec $\mathrm{H}_{\text {ext }}$ parallèle au plan des couches, l'échantillon étant incliné de $45^{\circ}$ par rapport au faisceau $\gamma$ incident. La variation de $X$ des pics intermédiaires du sextuplet principal traduit clairement la rotation des moments du fer dans le plan de l'échantillon. La figure 5 représente les variations de $X$, de $\tilde{\alpha}$ et de $H_{\text {hyp }}$ en fonction de $H_{\text {ext }}$. Cette évolution peut être interprétée comme suit: entre $H_{\text {ext }}=0$ (aimantation rémanente) et $H_{e x t}=2,5 \mathrm{kOe}, X$ reste aux alentours de 3,6 alors que $H_{\text {hyp }}\left(H_{\text {ext }}\right)$ augmente mettant en évidence un alignement presque total des moments du fer en sens inverse de $H_{\text {ext }}$. Pour $H_{\text {ext }}=2,5 \mathrm{kOe}, X$ diminue brusquement pour atteindre un minimum $X=1,45$ quand $H_{\text {ext }}=8,5$ kOe; les moments du fer sont en moyenne perpendiculaires à $H_{\text {ext }}$ et $H_{\text {hyp }}\left(H_{\text {ext }}\right)$ vaut sensiblement $H_{\text {hyp }}(0)$. 
Pour $\mathrm{H}_{\text {ext }}$ compris entre $8,5 \mathrm{kOe}$ et $13 \mathrm{kOe}, \mathrm{X}$ augmente mais $\mathrm{H}_{\text {hyp }}\left(\mathrm{H}_{\text {ext }}\right)$ est inférieur à $H_{\text {hyp }}(0)$ ce qui montre une tendance à l'alignement des moments ou fer dans le sens de $H_{\text {ext }}$. La valeur $H_{e x t}=2,5 \mathrm{kOe}$ correspond à $H^{*}$ déterminé à partir des mesures d'aimantation. On retrouve donc bien la transition état aligné-état twisté pour les moments du fer, directement mise en évidence au niveau microscopique.

\section{Conclusion}

Dans les multicouches $\mathrm{Fe} / \mathrm{Gd}$ les moments du fer sont confinés dans le plan des couches. Les isothermes magnétiques, les mesures de magnétoristance et la spectrométrie Mössbauer mettent clairement en évidence l'existence d'une transition "état aligné"-"état twisté" pour un champ critique $H^{*}$ appliqué parallèlement aux plans des couches du fer et du gadolinium dans le cas d'une multicouche $\mathrm{Fe}(42 \AA) / \mathrm{Gd}(84 \AA)$. La valeur de $H^{*}$ dépend de la température et de l'aimantation du fer et du gadolinium; l'état twisté est favorisé à la température de compensation, confirmant en cela les prévisions théoriques.

\section{Références}

[1] L. T. Baczewski, M. Piecuch, J. Durand, G. Marchal and P. Delcroix Phys. Rev. B 40 (1989) 11237

[2] Z. S. Shan and D. J. Sellmyer Phys. Rev. B 42 (1990) 10433

[3] K. Cherifi, C. Dufour, M. Piecuch, A. Bruson, Ph. Baver, G. Marchal and Ph. Mangin. EMRS Conference Proceedings (Strasbourg 1990) J. Mag. Mag. Mat. 93 (1991) 609

[4] T. Morishita, Y. Togami and K. Tsushima J. Phys. Soc. Japan 54 (1985) 37

[5] Y. Kamiguchi, Y. Hayakawa and H. Fujimori Appl. Phys. Lett. 55 (1989) 1918

[6] H. Fujimori, Y. Kamiguchi and H. Hayakawa J. Appl. Phys. 67 (1990) 5716

[7] R. E. Camley and D. R. Tilley

[8] R. E. Camley Phys. Rev. B 39 (1989) 12316

[9] C. Dufour et G.Marchal R. Sc. Ins. 62 (1991) 2984

[10] Ph. Bauer, M. Sajieddine, C. Dufour, K. Cherifi, G. Marchal et Ph. Mangin Europhys. Lett. 16 (1991) 307 